\title{
Summary
}

Our experiments dealing with diffusion through the excised cornea gave the following results :

1. Under the influence of colloidal osmotic forces a flow of water through the cornea is possible.

2. This permeability exists not only in the epithelial-endothelial direction, but also in the reverse direction.

3. Chloride ions under the influence of osmotic pressure can diffuse to the opposite surface in both directions.

\section{NEW DEVELOPMENTS IN THE PHARMACOLOGICAL TREATMENT OF PRIMARY GLAUCOMA}

BY

\author{
MARK J. Schoenberg, M.D. \\ NEW YORK
}

\section{Introduction}

IT is surprising that so little emphasis has been placed upon the significance of the fact that the pharmacological treatment of glaucoma consists, almost exclusively, of local applications of drugs which act principally upon the two subdivisions of the autonomic nervous system in the eye, the parasympathetic and sympathetic. This is probably one of the two reasons why the understanding of the mode of action of sympathomimetics (Sy-Mi) and parasympathomimetics $(\mathrm{Pa}-\mathrm{Sy}-\mathrm{Mi})^{*}$ upon the eyes has made little progress during past decades.

The other reason for this stagnation is that certain physiological data were missing. Without the knowledge of these data, no advance could be made. Recently, thanks to the theory of the chemical mediation of nerve impulses, the much-needed information has finally begun to become available. It has taken thirty

* Local applications of pilocarpine, eserine and of the new synthetic products. such as acetylcholine, mecholyl, prostigmin and doryl, have a stimulating action upon the ocular parasympathetic system and miosis is only one of several effects produced by these drugs. Atropine has an inhibitory or paralyzing effect upon the ocular parasympathetic system and mydriasis is only one of several reactions of the eye to this drug. It is, therefore, preferable to use the term, para-sympathomimetics (abbreviated: $\mathrm{Pa}-\mathrm{Sy}-\mathrm{Mi}$ ), instead of miotics or mydriatics, when speaking of these substances.

The same reasoning is applicable to drugs which stimulate, inhibit or paralyze the ocular sympathetic. They should be called sympatho-mimetics (abbreviated : Sy-Mi). 
years to lay the foundation of this new chapter of physiology and, although it is far from complete, it has already had a profound influence on our understanding of the physiology of the autonomic nervous system.

The object of this paper is twofold: (1) to submit some ideas concerning the mode of action of $\mathrm{Pa}-\mathrm{Sy}-\mathrm{Mi}$ and $\mathrm{Sy}-\mathrm{Mi}$ in normal and glaucomatous eyes; these ideas are based upon data discovered with the aid of the theory of chemical mediation of nerve impulses; (2) to suggest a programme of work still needed to fill in some of the gaps in our knowledge of the pharmacological treatment of glaucoma.

Before taking up this subject, it is necessary to make a short review of :

\section{The Essentials of the Theory of Chemical Mediation of Nerve Impulses*}

For a long time physiologists have been wondering how impulses travelling along nerves are being transmitted from the nerve terminals to secretory or muscle cells and by virtue of what device does the nerve impulse bring about the activity of a secretory or muscle cell. A beginning of the answer was given by Elliot in 1905.' He observed that a smooth muscle which contracts when the corresponding sympathetic nerve is stimulated, will also act after the complete degeneration of the nerve (artificially induced by extirpation or section), if epinephrin is applied to that muscle. Since the epinephrin could not act on the degenerated nerve terminals, it follows that its action was limited only to the muscle cells. Elliot concluded that impulses travelling along sympathetic nerves do not act directly upon the smooth muscle cells by " jumping " the neuromuscular partition. He

* The pharmacological studies which have uncovered data on chemical mediation of nerve impulses are numerous and it would be needless to review them in this paper. Those desiring to familiarize themselves with the subject will find an extensive bibliography in the papers of :

Butr, H. R.-Chemical Mediation of Nerve Impulses. Arch. Neurol. and Psychiat., Vol. XXXVII, p. 142 (January), 1937.

Myerson, A., and ThaU, W.-Human Autonomic Physiology. IX. Effect of Cholinergic and Adrenergic Drugs on the Eye. Arch. of Ophthal., Vol. LXXV, p. 78 (July), 1937.

DALE, Sir HENRY.- Transmission of Nervous Effects by Acetylcholine (Harvey Lectures, July, 1937). Bull. N. Y. Lcad. of Med., Vol. XIII, p. 380 (July), 1937.

LoEwi, OTto.-The Humoral Transmission of Nervous Impulse. (Harvey Lectures, 1932-1933; pp. 218-233.)

Newton, W. H.-Chemical Transmission at Nerve Endings. Recent Advances in Physiology, pp. 284-328, 1936.

Cannon, W. B.-Autonomous Neuro-effector Systems. New York, 1933.

1. Elliot, T. R.-The Action of Adrenalin. Jl. of Physiol., Vol. XXXII, pp. 401-467, 1905. 
assumed that on the arrival of the impulse at the nerve terminals an epinephrin-like substance (Sy) is " secreted" and that this chemical substance acts as the stimulus, bringing about the function of the muscle cells. This chemical substance is the chemical mediator of the sympathetic nerve impulses. Since 1905 a large number and a great variety of experiments were performed by physiologists and Elliot's experiments and conclusions were confirmed. The study of the transmission of nerve impulses to motile and secretory cells has been extended to the two components of the autonomic nervous system (the sympathetic and parasympathetic) and the fundamental discovery has been made-namely, that nerve impulses are transmitted to the secretory or contractile cells by the aid of a chemical mediator. The most important findings can be summarised as follows :

1. Impulses reaching the parasympathetic nerve terminals stimulate the formation of an acetylcholine-like substance (A-C), which acts upon the corresponding motile or secretory cells (effector cells).

2. Nerve impulses reaching the sympathetic nerve terminals release an epinephrin-like substance, called sympathin (Sy) which mediates the action of the impulse upon the corresponding effector cells.

3. The source of the chemical substance present at the neuroeffector junction from which the nerve impulse creates A-C is dependent for its maintenance on the integrity of the nerve endings. This source disappears or becomes depleted when the nerve fibres degenerate.

4. The A-C is destroyed by an esterase very soon after it has been produced. It is only during a short time, before it is neutralised, that A-C acts upon the effector cells. The process is repeated rhythmically as long as the nerve impulses arrive.

5. Eserine has the property of inhibiting the neutralising effect of esterase upon A-C.

6. Atropine prevents the effect of A-C.

7. Ergotoxin and ergotamin (gynergen) inhibit or paralyse the stimulating action of Sy on the effector cells.*

* Velhagen has demonstrated in the aqueous and tissue of the iris and retina a substance possessing all the properties of acetylcholine. He found that this substance acts directly on the sphincter muscle of the iris.

VelHaGen, K., Jr.-Cholin-Acetycholine as Physiologic Components of Retinal and Uveal Tissue. Arch.f. Augenheilk., Vol. CV, p. 573, 1932.

The Action of the Cholines on the Muscles of the Iris with Special Reference to their Double Innervation. Ibid., Vol. CVIII, p. 126, 1933.

Further Experiments on Active Principles in the Ocular Tissues. Ibid., Vol. CIX, p. 195, 1935. 


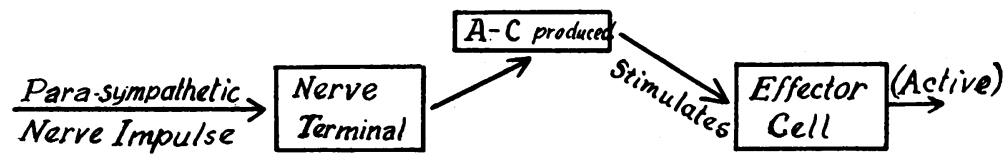

Figs l.a. PRxel-Sy neuro-effector unit in action.

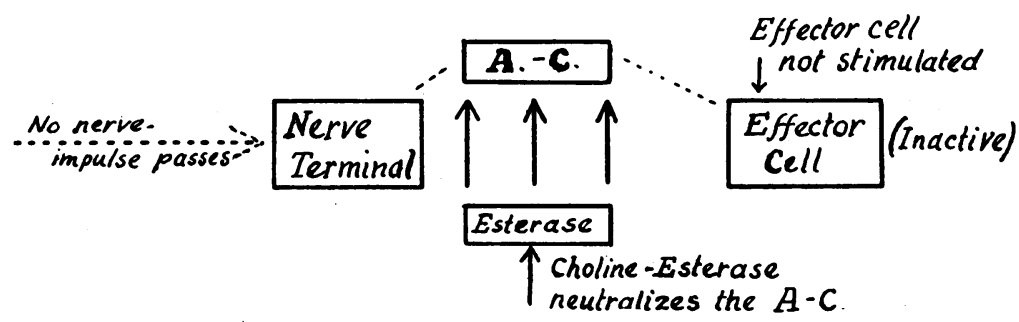

Fig 1.6. Parz-Sy neuro-effector unit
inactive

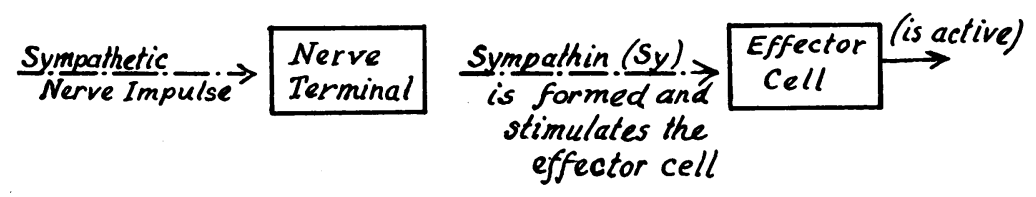
Fig. 2. Symporthetic neuro-effector unit in action.

We must keep in mind constantly that we are dealing with a unit, consisting of nerve terminals, effector cells, and the neuroeffector junction.

The implications of this theory are that the normal performances (release of A-C, esterase, contraction, relaxation, secretion) of the neuro-effector unit (setting the effector cells into action) depend on the following factors :

(a) the normal condition of the nerve fibres and nerve terminals;

(b) the "secretion" or release of normal amounts of A-C, or sympathin, respectively;

(c) the state of receptivity of the effector cells;

(d) the release at the parasympathetic nerve terminals of a normal amount of esteraze which neutralises the surplus of A-C. (No similar substance has been found for sympathin.) 
One may readily understand that in the eye, in which the two branches of the autonomic system are richly represented, a pathological process of the nerve fibres or their terminals, an increase or diminution of secretion of the chemical mediator (A-C or Sy), a reduction or an increase of sensitivity of the effector cells; or an increase or decrease in the quantity of esterase (at the nerve terminals) will bring about a disturbance of the function of the tissues innervated by one or of both branches of the autonomic nervous system.

\section{The Mode of Action of Pa-Sy-Mi in the Eye}

Having in mind the essential principles of the theory of chemical mediation of nerve impulses and the implications derived from them, we can better understand the mode of action of " mimetics" in the eye.

\section{The Pa-Sy-Mi}

The instillation in the eye of any one of the drugs, like - pilocarpine, eserine, prostigmin, acetylcholine and its derivatives (mecholyl and doryl)-has in many respects a similar effect : the pupillary and ciliary muscles contract, there is a relaxation of the zonula Zinii and, under favourable circumstances in glaucoma, the ocular tension is reduced. But, each one of these substances acts on a definite part of the neuro-effector unit.

Acetylcholine and its more stable derivates (mecholyl and doryl) are chemically quite similar to the A-C-like substance, which mediates the transmission of the parasympathetic nerve impulse. They act directly upon the effector cells just as if a nerve impulse, travelling along the nerve has released the chemical mediator (A-C). They act upon the effector cells even in the absence of a nerve impulse or when the intensity of the impulse is diminished.

The action of eserine and prostigmin is localised at the neuroeffector junction. It prevents the esterase from neutralising the A-C and, thereby, gives it a chance to act upon the effector cells (motile and secretory). Eserine cannot act directly upon the effector cells if no A-C is released.

Pilocarpine stimulates the effector cells, if they are chemically receptive.

In other words, the secretion of the ciliary processes and the contraction of the pupil and of the ciliary muscle, the change in the patency of blood vessels may be produced artificially by chemical substances which increase the amount of A-C, inhibit the action of esterase or stimulate directly the effector cells. These substances also increase the accommodation and tend to reduce the ocular tension. 
Scopolamine, atropine, and homatropine have an inhibitory paralysing effect, especially by rendering the corresponding effector cells insensitive to $A-C$. Practically, no matter how much eserine or A-C may be used in a thoroughly atropinised eye, the pupil will not contract nor will the ciliary body perform its function.

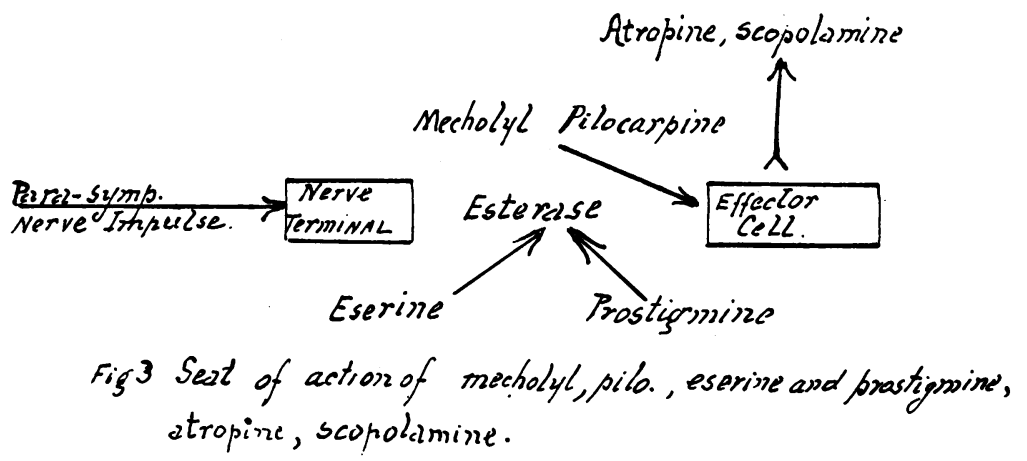

\section{The Si-Mi}

Epinephrin, ephedrin, benzedrin, ergotoxin or ergotamin tartrate act upon the sympathetic neuro-effector unit.

Epinephrin acts directly upon the effector cells. Its effect is similar to sympathin. It increases the size of the pupil, decreases slightly the accommodation, tends to raise the ocular tension and contracts blood vessels.

Cocaine is a vasoconstrictor and a dilator pupillae. It increases considerably the effect of epinephrin. Its action is on the sympathetic nerve terminals (?).

Ephedrine and benzedrin act somewhat similarly to epinephrin.

Ergotoxin and ergotamin tartrate (gynergen) inhibit the specific affinity of the effector cells for Sy. Their action on these cells is similar to that of atropine on the para-sympathetic effector cells.

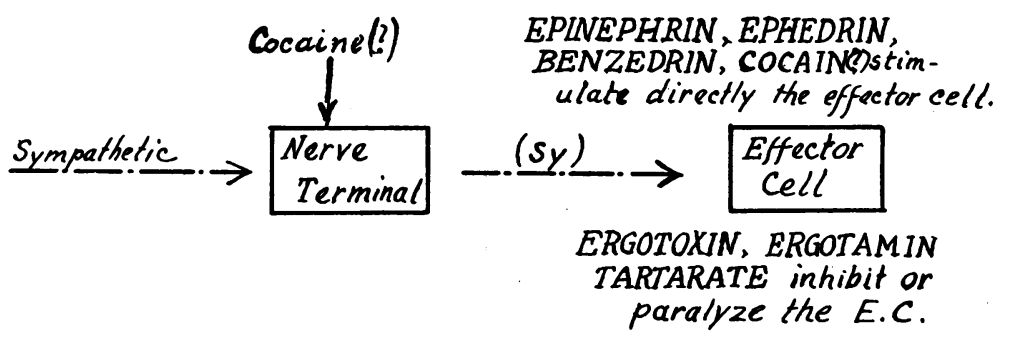

Fig 4. - Seat of action of Epinephrin, Ephedrix, benzedrix, cocain, and Ergatorite 


\section{The Rationale of the Pharmacological Local Treatment of Primary Glaucoma}

The rationale of the pharmacological treatment of glaucoma is based upon the assumption that the appearance of glaucoma signs and symptoms is in a certain measure related to a disturbance of the function of the autonomic nervous system in the eye. Some of the circumstantial evidence which speaks in favour of this assumption, one can enumerate :

(a) the effective action of some $\mathrm{Pa}-\mathrm{Sy}-\mathrm{Mi}$ and $\mathrm{Sy}-\mathrm{Mi}$ (with miotic action) in reducing the ocular hypertension and minimising other manifestations of glaucoma;

(b) the disastrous effect of some Pa-Sy-Mi and Sy-Mi (with mydriatic and cycloplegic action) in glaucoma, both groups of drugs being essentially $\mathrm{Pa}-\mathrm{Sy}-\mathrm{Mi}$, respectively Sy-Mi;"

(c) the specific response of the pupils to the instillation of epinephrin in eyes with prodromal glaucoma (the Knapp reaction);

(d) the fact that $\mathrm{Pa}-\mathrm{Sy}-\mathrm{Mi}$ do not reduce the ocular hypertension when administered by mouth or parenterally (in non-toxic doses), but diminish it when applied directly to the eye.

These evidences and the facts established with the aid of the theory of chemical mediation of nerve impulses, sketched in the first part of this paper, justify the conclusion that in glaucoma, one seat of disturbance is located in the neuro-effector unit of the autonomic nervous system within the eye.

Thus, we are led to think that at least for the present, in treating a case of primary glaucoma by pharmacological means, the first thing to do is to attempt to re-establish the normal function of the autonomic neuro-effector units within the eye.t Though groping in the dark, empiricism has done this very thing. It is significant that two drugs-eserine and pilocarpine-used against glaucoma during the past six decades, have a selective action upon the neuroeffector unit of the ocular autonomic nervous system. The recent additions to the pharmacological armamentarium against glaucoma (acetylcholine group, prostigmin and ergotoxin group), also have a specific affinity for and action upon the neuro-effector units.

Is our pharmacological armamentarium against primary glaucoma complete?

* One must admit that other changes besides blocking of the drainage system take place in the eye after using mydriatics and cycloplegics.

$\dagger$ This should not be interpreted to mean that these units are the only factor or the most important one involved in the causation of glaucoma. One can only say that besides surgery, the vegetative neuro-effector unit is, at the present time, the most accessible and responsive factor to our therapeutic efforts. 
Looking over the lists of drugs we realise its deficiencies and we are enabled-perhaps for the first time-to formulate the specific need for other drugs which :

(1) Stimulate or recondition the nerve endings of the parasympathetic fibres;

(2) Re-establish normal chemical processes that supply $A-C$;

(3) Reduce the over-production of esterase;

(4) Enhance the receptivity of the effector cells;

(5) Reduce the production of $S y$, and

(6) Diminish the over-sensitivity of the sympathetic effector cells.*

The problems are both clinical and pharmacological. It is probable that the diversity of responses of the glaucomatous eye to certain drugs depends in no small measure upon the location of the disturbance (whether predominantly in the parasympathetic or in the sympathetic; whether in the nerve terminals in the acetylcholine or sympathin formation area, or in the effector link of the neuro-effector unit. In the future, it will be the clinician's task to indicate whether the types are predominantly sympathetic, parasympathetic or mixed, and to point out which link or links of the neuro-effector units are involved in the various types or stages of the disease.

\section{Indications}

Pilocarpine and eserine, the drugs commonly employed in glaucoma, are indiscriminately used at present, either alone or in combination, without a definite indication. Since eserine prevents the neutralising effect of esterase on A-C and since it has no direct action upon the effector cells, it should be used in cases in which the amount of A-C is released in too small quantities or is being reduced by the over-production of esterase. Pilocarpine, on the other hand, acting directly upon the effector cells, is indicated when there is a deficiency in the function of these cells. Although we are as yet unable to distinguish between these two theoretical groups, the attempt has been made to separate them. (Case reports will be subject of a later communication.)

In cases in which there is a functional preponderance of the sympathetic component of the vegetative nervous system (with over-production of sympathin) or an enhanced sensitivity of the effector cells to Sy, drugs like ergotoxin or ergotamin tartrate,

* Walter B. Cannon (Proceedings of the Staff Meetings of the Mayo Clinic, Vol. XIII, January 19,1938 ) has recently made the following statement: "It would appear that there may be a general law that denervation increases the sensitiveness of structures to stimulation." This statement is based upon a number of reliable experimental data. 
Pharmacological Treatment of Primary Glaucoma 425

which have a depressing or paralysing effect upon the sympathetic effector cells, are indicated. In such cases, A-C, pilocarpine and eserine may act as an adjuvant, but cannot succeed alone in correcting the condition.

In mixed forms, such as a failing parasympathetic and an overacting sympathetic, drugs that enhance the function of the first and depress that of the second have a better chance of success.

\section{Summary}

1. Nerve impulses travelling along the two branches of the vegetative nervous system are transmitted to the secretory or contractile cells by the aid of a chemical mediator (acetylcholine or sympathin, respectively).

2. There are evidences which support the assumption that in primary glaucoma, there is a disturbance (functional or organic) of one or both branches of the vegetative nervous system, within the eye.

3. The present pharmacological treatment of primary glaucoma endeavours to correct such a disturbance by dealing with each or with all three of the components of the neuro-effector unit.

4. As far as we know, drugs that have a beneficial effect upon eyes with primary glaucoma, possess the property of acting either on the esterase (which neutralises acetylcholine) or on the effector cells.

5. There is need of more knowledge on the subject of dysfunction or degeneration of the parasympathetic and sympathetic nerve terminals, more information on details regarding the process of release of acetylcholine and esterase, also on affections of effector cells. There is also need of drugs or other means which can re-establish the normal function of any one of the three crmponents of the neuro-effector unit, whenever impaired.

6. Clinical experience leads us to believe that in some cases of primary glaucoma, we are dealing with a failing function of the ocular parasympathetic, in others with a preponderance of the ocular sympathetic, and in still other cases with a combination of both conditions. Thus, we may classify-for the present-the various types of primary glaucoma in three groups : sympathetic, parasympathetic and mixed.

$\tau$. The adoption of this classification may prove to be helpful to the progress of our knowledge of the pharmacological treatment of primary glaucoma.

Thanks are due to the firms of Hoffman La Roche, Sandoz and Merck for furnishing prostigmin, gynergen and mecholyl for trial and study. 